\title{
Impact of pump wavelength on terahertz emission of a cavity-enhanced spintronic trilayer
}

Cite as: Appl. Phys. Lett. 114, 041107 (2019); https://doi.org/10.1063/1.5048297

Submitted: 12 July 2018 . Accepted: 21 December 2018 . Published Online: 01 February 2019

R. I. Herapath (D), S. M. Hornett (D), T. S. Seifert (D), C. Jakob (D), M. Kläui (D), J. Bertolotti (D), T. Kampfrath, and E. Hendry

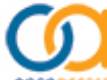




\title{
Impact of pump wavelength on terahertz emission of a cavity-enhanced spintronic trilayer
}

\author{
Cite as: Appl. Phys. Lett. 114, 041107 (2019); doi: 10.1063/1.5048297 \\ Submitted: 12 July 2018 - Accepted: 21 December 2018 · Published Online: \\ 01 February 2019
}

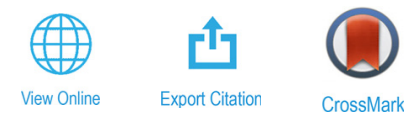

R. I. Herapath,, ${ }^{1, a)}$ (D S. M. Hornett, ${ }^{7}$ (D) T. S. Seifert, ${ }^{2,3}$ (D) G. Jakob,, ${ }^{4,5}$ (D) M. Kläui, ${ }^{4,5}$ (D) J. Bertolotti, ${ }^{7}$ (D) T. Kampfrath, ${ }^{2,3}$
and E. Hendry

\section{AFFILIATIONS}

${ }^{7}$ University of Exeter, Physics Building, Stocker Road, Exeter EX4 4QL, United Kingdom

${ }^{2}$ Fritz Haber Institute of the Max Planck Society, 14195 Berlin, Germany

${ }^{3}$ Department of Physics, Freie Universität Berlin, 14195 Berlin, Germany

${ }^{4}$ Institute of Physics, Johannes Gutenberg University Mainz, 55099 Mainz, Germany

${ }^{5}$ Graduate School of Excellence Materials Science in Mainz, Staudingerweg 9, 55128 Mainz, Germany

a) rh522@exeter.ac.uk

\begin{abstract}
We systematically study the pump-wavelength dependence of terahertz pulse generation in thin-film spintronic THz emitters composed of a ferromagnetic CoFeB layer between adjacent nonmagnetic W and Pt layers. We find that the efficiency of THz generation is essentially flat for excitation by $150 \mathrm{fs}$ pulses with center wavelengths ranging from 900 to $1500 \mathrm{~nm}$, demonstrating that the spin current does not depend strongly on the pump photon energy. We show that the inclusion of dielectric overlayers of $\mathrm{TiO}_{2}$ and $\mathrm{SiO}_{2}$, designed for a particular excitation wavelength, can enhance the terahertz emission by a factor of up to two in field.

(c) 2019 Author(s). All article content, except where otherwise noted, is licensed under a Creative Commons Attribution (CC BY) license (http://creativecommons.org/licenses/by/4.0/). https://doi.org/10.1063/1.5048297
\end{abstract}

Terahertz $(\mathrm{THz})$ radiation is non-ionizing and, therefore, safe for many applications, ranging from micro- and macroscopic imaging and spectroscopy to wireless communication. ${ }^{1-4}$ However, the spectroscopically interesting $\mathrm{THz}$ frequency band near $1 \mathrm{THz}$ is not easily accessible. Electronic sources such as oscillators can only provide high (milliwatt) output levels up to a few $100 \mathrm{GHz},{ }^{5}$ while optical sources such as quantum cascade lasers are typically limited to frequencies $>2 \mathrm{THz}$ at room temperature. ${ }^{4,6}$ To fill this "gap," considerable effort has been garnered towards sources capable of frequency mixing and optical rectification, ${ }^{7}$ typically driven by femtosecond lasers.

To date, most $\mathrm{THz}$ emitting materials have been found to be insulators or semiconductors. ${ }^{8}$ Recently, THz emitters based on magnetic, metallic thin films have been demonstrated which emit $\mathrm{THz}$ radiation under illumination by femtosecond pulses. $^{9-15}$ We here focus on trilayer thin-film emitters formed from a ferromagnetic (FM) layer between two nonferromagnetic (NM) layers. A two-step process is thought to generate $\mathrm{THz}$ radiation: ${ }^{16}$ Upon excitation by the femtosecond pump pulse, an ultrashort out-of-plane spin current polarized along the FM magnetization is injected from the FM into the NM layers. Thereafter, the inverse spin Hall effect converts the laser-induced spin current into a transverse in-plane charge current within the NM layer which leads to the emission of a THz pulse into the optical far-field. ${ }^{16-18}$

One of the most efficient films of this type ${ }^{9}$ comprises $\mathrm{W}$, $\mathrm{CoFeB}$, and Pt layers. Importantly, Pt and W feature a spin Hall angle of opposite sign, resulting in a constructive superposition of the two charge currents in these layers. The result is an ultrabroadband $\mathrm{THz}$ emitter, capable of delivering pulses spanning 0.1 to $30 \mathrm{THz} .{ }^{9}$ With an active region only a few nanometers thick in total, these emitters can generate as much $\mathrm{THz}$ amplitude as a phase-matched electro-optic crystal of millimeter thickness. ${ }^{9}$ Such highly efficient, but thin, THz emitters show much promise, particularly for near-field measurement or applications that benefit from the absence of phase matching.

Most studies of these $\mathrm{THz}$ emitters have been carried out using Ti:sapphire laser sources with wavelengths around $800 \mathrm{~nm}$. However, many thin metal films show a rather wavelengthindependent absorptance in the visible and near infrared, such that the $\mathrm{THz}$-generation efficiency may naively be expected to be 
largely independent of the pump wavelength. ${ }^{9}$ Such wavelengthindependent emission would be a great advantage of these types of emitters, allowing users free choice in excitation laser sources. Such a feature is particularly interesting in terms of fiber-based lasers that operate at infrared wavelengths above $1 \mu \mathrm{m}$.

In this paper, we investigate the pump-wavelength dependence of $\mathrm{THz}$ emission of $\mathrm{W}|\mathrm{CoFeB}| \mathrm{Pt}$ trilayer emitters using a continuously tunable femtosecond laser source. We find that the efficiency of THz generation is surprisingly flat for excitation by 150 fs pulses with central wavelengths ranging from 900 to $1500 \mathrm{~nm}$. This observation reveals that the photon energy has little effect on the number of electrons contributing to transport of spin polarization and that the key parameter is the total amount of energy deposited by the pump pulse. We demonstrate that the inclusion of dielectric overlayers $\left(\mathrm{TiO}_{2}\right.$ and $\mathrm{SiO}_{2}$ ), forming a cavity with the substrate, can enhance emission by up to a factor of four in intensity in the frequency window from 0 to $2 \mathrm{THz}$. This optimized emitter can be readily used in setups driven by fiber-based systems with wavelengths around $1030 \mathrm{~nm}$.

A schematic of the samples is shown in Fig. 1. The emitters have each $10 \mathrm{~mm}$ by $7 \mathrm{~mm}$ in area, with layers of $\mathrm{W}, \mathrm{CoFeB}$, and Pt deposited on a sapphire substrate by Ar sputtering using a Singulus Rotaris ${ }^{\circledR}$ deposition system. ${ }^{9}$ The layers of $\mathrm{W}, \mathrm{CoFeB}$, and Pt have nominal thicknesses of $2 \mathrm{~nm}, 1.8 \mathrm{~nm}$, and $2 \mathrm{~nm}$, respectively, giving a total thickness of $5.8 \mathrm{~nm}$ for the metallic layers. On six samples, an optical cavity made from alternating dielectric overlayers of $\mathrm{TiO}_{2}$ (thickness $113 \mathrm{~nm}$ and near-infrared index of refraction 2.265$)^{19}$ and $\mathrm{SiO}_{2}(185 \mathrm{~nm}, 1.455)$, with a total overlayer thickness of $1.495 \mu \mathrm{m}$, was deposited by plasmaassisted electron beam evaporation using a Leybold 1104 coating platform. Note that the overlayer thicknesses were optimized

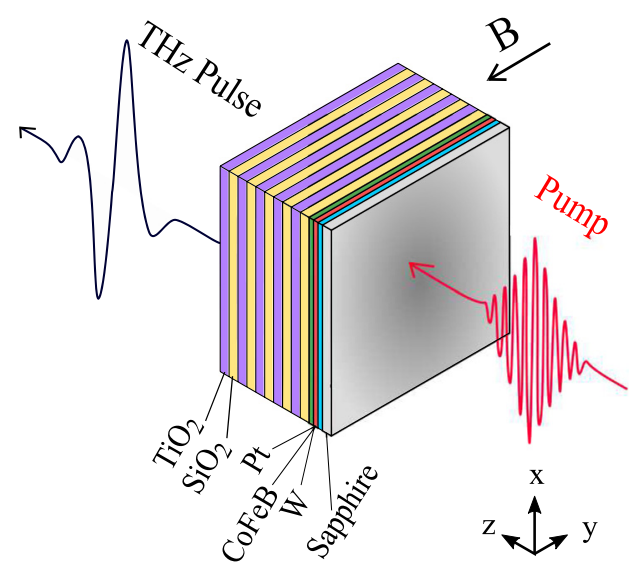

FIG. 1. Schematic of a spintronic trilayer with added dielectric cavity, grown on $0.5 \mathrm{~mm}$ of sapphire $\left(\mathrm{Al}_{2} \mathrm{O}_{3}\right)$. The near-infrared pump pulse, incident through the substrate, is partially absorbed in the metallic layers, launching a spin current from the ferromagnetic (FM) layer into the nonmagnetic (NM) layers. The inverse spin Hall effect converts this ultrashort out-of-plane spin current into an in-plane charge current resulting in the emission of $\mathrm{THz}$ radiation into the optical far-field. A weak in-plane magnetic field $(B)$ determines the magnetization direction and the linear polarization of the emitted $\mathrm{THz}$ field. numerically to give a flat response at the target wavelength. The near-infrared transmission spectrum, normalized by the optical transmission of the sapphire substrate, was recorded for each sample using a monochromator (Oriel) and a Quartz TungstenHalogen lamp (Thorlabs) [see Figs. 2(a) and 2(b)].

$\mathrm{THz}$ emission was investigated using wavelength-tunable near-infrared pump pulses (duration $\sim 150 \mathrm{fs}$, spot diameter $\sim 4 \mathrm{~mm}$, and fluence $\sim 0.1 \mathrm{~J} \mathrm{~m}^{-2}$ ) incident normal to the sample surface. They were generated by an optical parametric amplifier (Light Conversion TOPAS) driven by an $800 \mathrm{~nm}$ Ti:Sapphire amplified laser ( $\sim 100 \mathrm{fs}$, repetition rate of $1 \mathrm{kHz}$ ). When varying the pump wavelength, we paid particular attention to keeping the other pump-pulse parameters, in particular, energy, duration, and focus diameter, constant; we worked far from the focal point of our lens to maintain the spot diameter. The resulting $\mathrm{THz}$ pulse, emitted in the forward direction, is detected through electro-optic sampling with $800 \mathrm{~nm}$ pulses from our amplified laser system using a $1 \mathrm{~mm}$ thick, (110)-oriented ZnTe crystal. For all samples, a neodymium magnet attached to the emitter holder approximately $7 \mathrm{~mm}$ from the edge of the film gives an in-plane magnetic field of $\sim 13 \mathrm{mT}$, as depicted in Fig. 1. This determines the linear polarization of the emitted $\mathrm{THz}$ electric field which is always oriented perpendicularly to the magnetic field.

Figure 2(c) shows a typical electro-optic signal waveform of a THz pulse emitted from the bare spintronic trilayer after excitation by a pump pulse with a center wavelength of $1040 \mathrm{~nm}$. The signal amplitude grows approximately linearly for incident pump fluences at least up to $0.4 \mathrm{~J} \mathrm{~m}^{-2}$ (see supplementary material Fig. S1).

In Fig. 2(e), we plot the integrated $\mathrm{THz}$ field emitted by a $\mathrm{W}|\mathrm{CoFeB}| \mathrm{Pt}$ trilayer sample for various pump wavelengths in the range from 900 to $1500 \mathrm{~nm}$. Remarkably, over this range, the efficiency of $\mathrm{THz}$ generation is wavelength-independent. Importantly, since they originate from the same source, the focus diameter, energy, and duration of the pump pulses are fairly independent of the wavelength in the range of 1000 to $1300 \mathrm{~nm}$. For example, the pulse duration varies by less than $12 \mathrm{fs}$ across this entire range (see supplementary material Figs. S2 and supplementary material Sec. B). An unchanged THz signal amplitude has also been reported ${ }^{12}$ for $1550 \mathrm{~nm}$ and the significantly shorter wavelength of $800 \mathrm{~nm}$. Our results and those of Ref. 12 indicate that the spin current arises from the hot electrons induced by the pump pulse. The details of the involved optical transitions are insignificant. Therefore, the key parameter is the amount of energy that is deposited by the pump pulse in the electronic system.

Since the $\mathrm{THz}$ emission from the spintronic emitters is largely independent of the pump wavelength, one can in principle enhance the emission for any particular wavelength by designing a suitable adjacent cavity (Fig. 1). A straightforward implementation is dielectric overlayers similar to a Bragg mirror, forming a broadband dielectric cavity with the substrate. By placing the lower index material, in this case $\mathrm{SiO}_{2}$, next to the trilayer, an intensity maximum is formed within the active $\mathrm{THz}$ generation layers. Doing so, for a particular target pump 

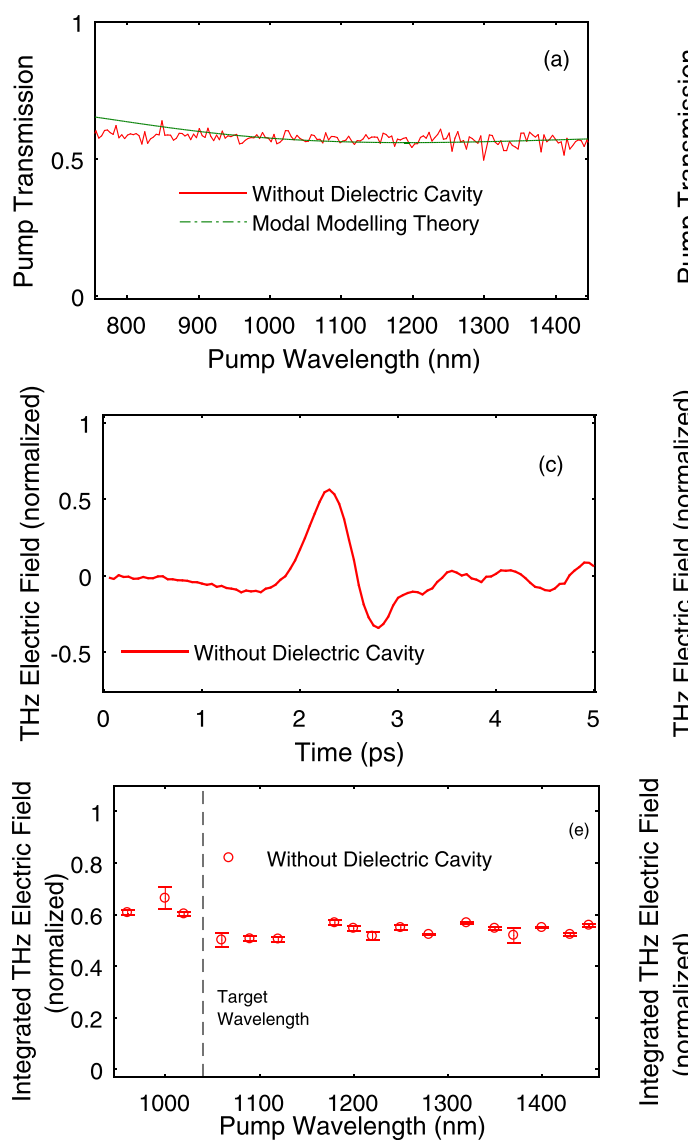
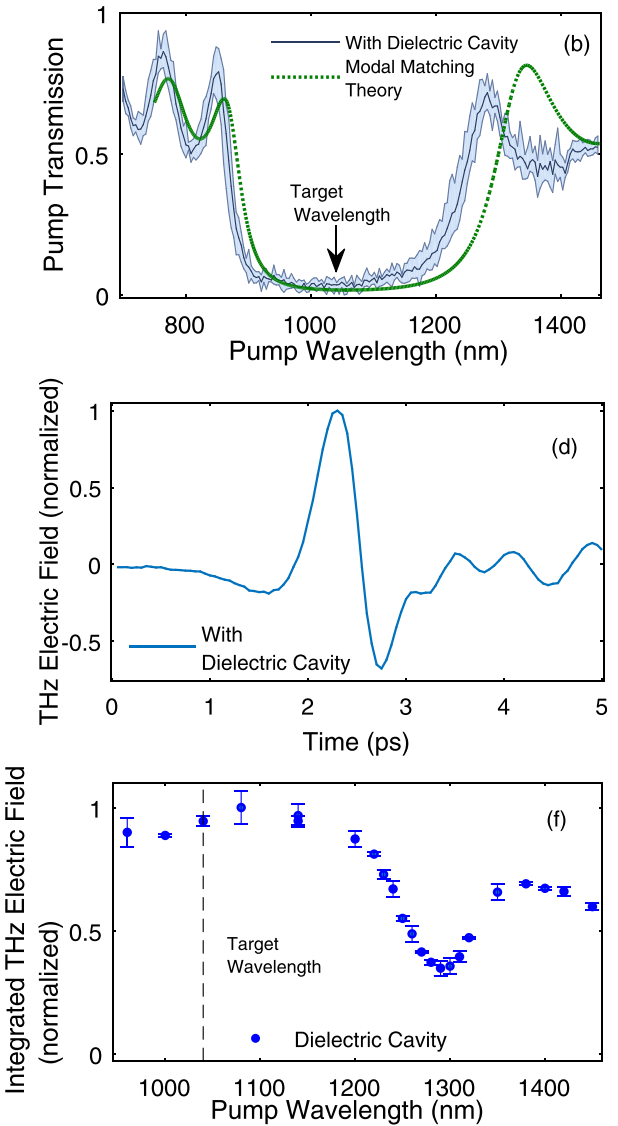

FIG. 2. (a) and (b) Measured transmittance of samples without (left) and with (right) $\mathrm{TiO}_{2}$ and $\mathrm{SiO}_{2}$ dielectric overlayers. The blue-shaded region in panel (b) represents variation among the six cavity films due to variation in the fabrication process. Whilst the transmittance of the trilayer alone is relatively flat with the incident wavelength, the trilayer with the dielectric overlayers exhibits a bandstop region between 900 and $1200 \mathrm{~nm}$. Green lines are calculated using modal matching (see supplementary material). (c) and (d) Typical $\mathrm{THz}$ waveforms from the bare trilayer sample (left) and the trilayer with overlayers (right) pumped by $1040 \mu \mathrm{m}$ pulses. (e) and (f) The THz electric field, integrated over the spectrum, emitted without (left) and with (right) dielectric overlayers as a function of the pump wavelength while the energy, focus diameter, and duration of the pump pulses were kept constant. Error bars represent variation in emission between nominally identical samples. Note that the highest value of the $\mathrm{THz}$ field has been normalized to one to ease comparison. wavelength, pump absorption in the metallic trilayer will be maximized and so will the emitted $\mathrm{THz}$ radiation. For convenience, we choose a target pump wavelength of $1040 \mathrm{~nm}$ aimed at common fiber lasers, specified by the thickness and type of the dielectric layers. To achieve a stop-band with less than 5\% transmission, a total of 10 dielectric layers are required. For these coated samples, the total thickness (neglecting the substrate) is $1.495 \mu \mathrm{m}$, corresponding to an optical thickness for THz wavelengths of approximately $9 \mu \mathrm{m}$, which is still very subwavelength for $\mathrm{THz}$ radiation.

To model the behavior of the emitter with a dielectric cavity, a standard modal matching approach ${ }^{20}$ is used which can also treat a source within the active region (see supplementary material Sec. D). In Fig. 2(b), we plot the calculated near-infrared transmission spectrum for the samples with dielectric overlayers (green dotted-dashed line). We expect a transmittance minimum [Fig. 2(b)] and an absorptance maximum (see supplementary material Sec. D) at the target wavelength of $1040 \mathrm{~nm}$, with a bandwidth of around $300 \mathrm{~nm}$.

In Fig. 2(b), the blue-shaded region represents the distribution of transmittance spectra measured for the six samples with overlayers. While the transmittance [Fig. 2(b)] and absorptance (supplementary material Fig. S4) of the sample without the dielectric overlayers are fairly flat over the measured range, all samples with dielectric overlayers show clear band-stop behavior. The minimum transmittance in the measurement, $\sim 3 \%$, is consistent with our modeling. The slight shift in the wavelength of the band-stop region between the modeling and experiment likely arises from variation in the refractive indices and/or thicknesses of the dielectric layers. For example, the optical thickness needs to change by only $3.8 \%$ with respect to specified values to account for this discrepancy. The band-stop in the region of the target wavelength designates a region where we expect to see enhancement of $\mathrm{THz}$ emission.

In Fig. 2(f), we can observe the enhancement in THz emission generated by the dielectric overlayers. For an identical pump fluence, in the band-stop window (900 to $1200 \mathrm{~nm}$ ), we find a $\mathrm{THz}$ field that is a factor of $\sim 2$ (intensity of a factor $\sim 4$ ) larger compared to the trilayer without overlayers. This is slightly larger than the $70 \%$ enhancement in field recently reported for a cavity formed from two sets of spintronic layers separated by dielectric spacers. ${ }^{13}$ We also observe reduction in $\mathrm{THz}$ emission near the near-infrared transmittance maxima at $1300 \mathrm{~nm}$. In these bandpass regions, the dielectric overlayers act to reduce the near-infrared intensity in the active magnetic layer. For the band-stop region, the factor of two enhancement in $\mathrm{THz}$ field emission is observed for the entire fluence range investigated here (supplementary material Fig. S1). 


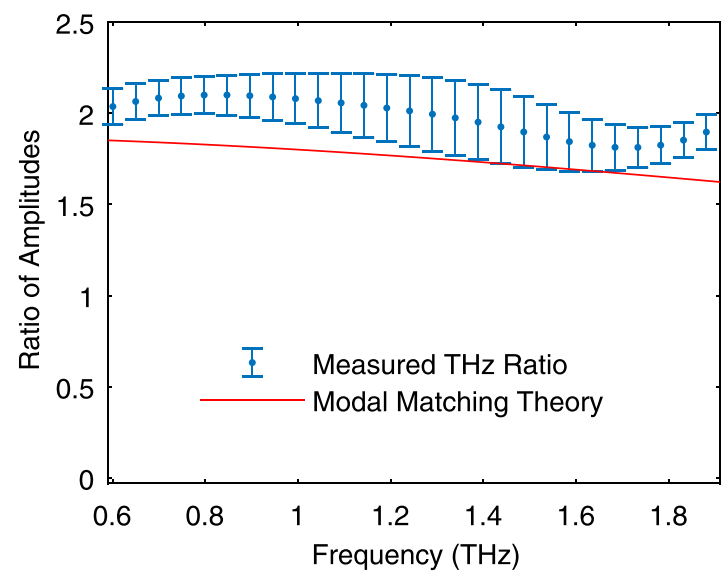

FIG. 3. Measured THz emission enhancement for different THz spectral components, plotting the $\mathrm{THz}$ field amplitude emitted from a spintronic trilayer with the dielectric cavity divided by field emitted without cavity, when excited by a pump pulse of $1040 \mathrm{~nm}$. The solid line is a calculation from modal matching theory.

In Fig. 3, we plot the spectral dependence of this observed $\mathrm{THz}$ emission enhancement for excitation by a $1040 \mathrm{~nm}$ pump pulse (blue dots). The highest enhancement is observed for the lowest $\mathrm{THz}$ frequencies. Above $1 \mathrm{THz}$, we begin to see a slight drop in the field enhancement factor. The most significant limitation within the experiment is the bandwidth of the ZnTe detector, which limits our detection frequencies to $<2 \mathrm{THz}$. However, after normalising the emission, thus removing the effects of the detector response function, absorption manifests itself as a reduction in the field enhancement factor for frequencies $>1 \mathrm{THz}$. Also shown is the prediction from modeling (red line): this is calculated from the modal modeling method using the $\mathrm{THz}$ field emitted with and without the dielectric cavity, multiplied by the predicted pump intensity in the active magnetic layer. Our modeling predicts a similar behavior, but with an enhancement factor which decreases more quickly with frequency, an effect which arises due to the frequency dependent absorption of $\mathrm{TiO}_{2}$. In our model, we also observe high sensitivity to the precise index of the Pt layer, a material which shows high variation dependent on the morphology. ${ }^{21}$ It is important to note that alternative dielectrics to $\mathrm{TiO}_{2}$ may well exhibit lower absorption losses and could increase the operational bandwidth of cavity enhanced emitters.

To conclude, we systematically study the pump-wavelength dependence of $\mathrm{THz}$ emission of spintronic $\mathrm{W}|\mathrm{CoFeB}| \mathrm{Pt}$ trilayers. We find that the efficiency of THz generation is essentially flat for excitation by 150 fs pulses with central wavelengths ranging from 900 to $1500 \mathrm{~nm}$, indicating that the spin current is largely independent of the pump-photon energy. We demonstrate that the inclusion of dielectric overlayers of $\mathrm{TiO}_{2}$ and $\mathrm{SiO}_{2}$, designed for a particular excitation wavelength, can enhance emission by up to a factor of two in field amplitude in this technologically important range of pump wavelengths. The four-fold enhancement in emitted $\mathrm{THz}$ intensity could be further improved using cavities with higher quality factors, matched to the bandwidth of the pump pulse. As shown for pump wavelengths at around
$800 \mathrm{~nm}$, shorter pump pulses in the infrared are expected to deliver more broadband THz transients, reaching frequencies of up to $30 \mathrm{THz}$ for pump durations approaching $10 \mathrm{fs}$.

See supplementary material for a power dependence of the spintronic trilayer with and without the dielectric cavity, the dependence of the pump-pulse duration on the wavelength, the detector response function of our $\mathrm{THz}$ electro-optic detection system, and details on the modal matching theory used for the modeling. ${ }^{22-28}$

The authors like to acknowledge support via the EPSRC Centre for Doctoral Training in Metamaterials (Grant No. EP/L015331/1). E.H. acknowledges support from EPSRC fellowship (EP/K041215/1). T.K., T.S.S., M.K., and G.J. acknowledge the German Research Foundation for funding through the collaborative research centers SFB TRR 227 Ultrafast spin dynamics (Project B02) and SFB TRR 173 Spin $+\mathrm{X}$ as well as the Graduate School of Excellence Materials Science in Mainz (MAINZ, GSC 266). TK also acknowledges funding through the ERC H2020 CoG project TERAMAG/ Grant No. 681917.

\section{REFERENCES}

${ }^{1}$ A. Y. Pawar, D. D. Sonawane, K. B. Erande, and D. V. Derle, Drug Invent. Today 5, 157 (2013).

${ }^{2}$ J. F. Federici, B. Schulkin, F. Huang, D. Gary, R. Barat, F. Oliveira, and D. Zimdars, Semicond. Sci. Technol. 20, S266 (2005).

${ }^{3}$ F. Sizov and A. Rogalski, Prog. Quantum Electron. 34, 278 (2010).

${ }^{4}$ S. S. Dhillon, M. S. Vitiello, E. H. Linfield, A. G. Davies, M. C. Hoffmann, J. Booske, C. Paoloni, M. Gensch, P. Weightman, G. P. Williams, E. CastroCamus, D. R. S. Cumming, F. Simoens, I. Escorcia-Carranza, J. Grant, S. Lucyszyn, M. Kuwata-Gonokami, K. Konishi, M. Koch, C. A. Schmuttenmaer, T. L. Cocker, R. Huber, A. G. Markelz, Z. D. Taylor, V. P. Wallace, J. Axel Zeitler, J. Sibik, T. M. Korter, B. Ellison, S. Rea, P. Goldsmith, K. B. Cooper, R. Appleby, D. Pardo, P. G. Huggard, V. Krozer, H. Shams, M. Fice, C. Renaud, A. Seeds, A. Stöhr, M. Naftaly, N. Ridler, R. Clarke, J. E. Cunningham, and M. B. Johnston, J. Phys. D: Appl. Phys. 50, 043001-043049 (2017).

${ }^{5} \mathrm{X}$. C. Zhang and J. Xu, Introduction to THz Wave Photonics (Springer, 2010), pp. 1-246.

${ }^{6}$ R. A. Lewis, J. Phys. D: Appl. Phys. 47, 4 (2014).

${ }^{7}$ N. M. Burford and M. O. El-Shenawee, Opt. Eng. 56, 010901 (2017).

${ }^{8}$ Y.-S. Lee, Principles of Terahertz Science and Technology (Springer US, Boston, MA, 2009).

${ }^{9}$ T. Seifert, S. Jaiswal, U. Martens, J. Hannegan, L. Braun, P. Maldonado, F. Freimuth, A. Kronenberg, J. Henrizi, I. Radu, E. Beaurepaire, Y. Mokrousov, P. M. Oppeneer, M. Jourdan, G. Jakob, D. Turchinovich, L. M. Hayden, M. Wolf, M. Münzenberg, M. Kläui, and T. Kampfrath, Nat. Photonics 10, 483 (2016).

${ }^{10}$ T. J. Huisman and T. Rasing, J. Phys. Soc. Jpn. 86, 011009 (2017).

${ }^{1}$ D. Yang, L. Jianhui, Z. Chao, S. Lu, Z. Ronger, L. Shenhnian, Y. Wu, J. Qi, B. Lakshmi, I. M. Hall, C. Egan, J. Alexander, L. Zender, M. S. Spector, W. Xue, S. W. Lowe, M. Wigler, and R. Lucito, Adv. Opt. Mater. 4, 1944 (2016).

${ }^{12}$ E. T. Papaioannou, G. Torosyan, S. Keller, L. Scheuer, M. Battiato, V. Katrine, L. Johannes, M. Tani, and R. Beigang, IEEE Trans. Magn. 54(11), 1 (2018).

${ }^{13}$ Z. Feng, R. Yu, Y. Zhou, H. Lu, W. Tan, H. Deng, Q. Liu, L. Zhu, J. Cai, B. Miao, and H. Ding, Adv. Opt. Mater. 6(23), 1800965 (2018).

${ }^{14}$ G. Torosyan, S. Keller, L. Scheuer, R. Beigang, and E. T. Papaioannou, Sci. Rep. 8, 1311 (2018).

${ }^{15}$ Y. Wu, M. Elyasi, X. Qiu, M. Chen, Y. Liu, L. Ke, and H. Yang, Adv. Mater. 29, 1603031 (2017). 
${ }^{16}$ T. Kampfrath, M. Battiato, P. Maldonado, G. Eilers, J. Nötzold, S. Mährlein, V. Zbarsky, F. Freimuth, Y. Mokrousov, S. Blügel, M. Wolf, I Radu, P. M. Oppeneer, and M. Münzenberg, Nat. Nanotechnol. 8, 256 (2013).

${ }^{17}$ M. Battiato, K. Carva, and P. M. Oppeneer, Phys. Rev. Lett. 105, 027203 (2010).

${ }^{18}$ E. Saitoh, M. Ueda, H. Miyajima, and G. Tatara, Appl. Phys. Lett. 88, 182509 (2006).

${ }^{19}$ Refractive index values at $1040 \mathrm{~nm}$ obtained via private communication from artemis optical.

${ }^{20}$ M. S. Tomas, Phys. Rev. A 51, 2545 (1995).

${ }^{21}$ S. A. Kovalenko and M. P. Lisitsa, "Semiconductor physics," Quantum Electron. Optoelectron. 4, 352 (2001).
${ }^{22}$ D. Saeedkia, in Proceedings of the 5th European Conference on Antennas and Propagation (EUCAP) (2011), p. 3326.

${ }^{23} \mathrm{~J}$. Shan and T. F. Heinz, Ultrafast Dynamical Processes in Semiconductors, Topics in Applied Physics Series (Springer, Berlin, 2004), Vol. 92, pp. 1-56.

${ }^{24}$ X. Zheng, A. Sinyukov, and L. M. Hayden, Appl. Phys. Lett. 87, 081115 (2005).

${ }^{25}$ D. Grischkowsky, S. Keiding, M. van Exter, and C. Fattinger, J. Opt. Soc. Am. B 7, 2006 (1990).

${ }^{26}$ J. Zak, E. R. Moog, C. Liu, and S. D. Bader, J. Magn. Magn. Mater. 89, 107 (1990). ${ }^{27}$ T. Kampfrath, J. Nötzold, and M. Wolf, Appl. Phys. Lett. 90, 231113 (2007).

${ }^{28}$ V.-S. Dang, H. Parala, J. H. Kim, K. Xu, N. B. Srinivasan, E. Edengeiser, M. Havenith, A. D. Wieck, T. de los Arcos, R. A. Fischer, and A. Devi, Phys. Status Solidi (a) 211, 416 (2014). 